Trigonometric Bézier and

Stancu Polynomials

Guido Walz

Nr. 217/1996 


\title{
Trigonometric Bézier and Stancu Polynomials over Intervals and Triangles
}

\author{
Guido Walz
}

Abstract. We introduce a family of trigonometric polynomials, denoted as Stancu polynomials, which covers as special cases the trigonometric Lagrange and Bernstein polynomials. This family depends only on one real parameter, denoted as design parameter. Our approach works for curves as well as for surfaces over triangles. The resulting Stancu curves resp. surfaces therefore establish a link between trigonometric interpolatory and Bernstein-Bézier curves resp. surfaces.

Keywords. Trigonometric Lagrange polynomials, trigonometric Bernstein polynomials, Stancu polynomials, design parameter.

Guido Walz

Fakultät für Mathematik und Informatik

Universität Mannheim

D-68131 Mannheim

Germany

walz@math.uni-mannheim.de 


\section{Introduction}

Recently, trigonometric splines and polynomials have gained very much interest within CAGD, in particular curve design, see for example Alfeld, Neamtu, Schumaker (1995), Koch, Lyche, Neamtu, Schumaker (1995), Gonsor \& Neamtu (1996), Peña (1996), Walz (1997). The aim of the present note is twofold: First, similarly as done in the (algebraic) polynomials case in Farin \& Barry (1986) and Walz $(1988,1991)$, we introduce a one-parameter family of trigonometric polynomials, which provides a link between the trigonometric Lagrange polynomials (1.1) and Bernstein polynomials (1.2). Then, in section 2, this approach will be transfered to trigonometric polynomials (in barycentric coordinates) on a triangle.

\section{Stancu Polynomials on an Interval}

We consider, for even integer $n$, the $(n+1)$-dimensional space of trigonometric polynomials

$$
\mathcal{T}_{m}:=\operatorname{span}\{1, \cos (2 \tau), \sin (2 \tau), \ldots, \cos (n \tau), \sin (n \tau)\}
$$

where $0 \leq \tau \leq 1$. The Lagrange polynomials (w.r.t. equidistant nodes) from $\mathcal{T}_{m}$ are the functions

$$
l_{\nu}(\tau)=\prod_{\substack{j=0 \\ j \neq \nu}}^{n} \frac{\sin \left(\tau-\frac{j}{n}\right)}{\sin \left(\frac{\nu-j}{n}\right)}, \nu=0, \ldots, n,
$$

whereas the corresponding Bernstein polynomials are

$$
b_{\nu}(\tau)=\frac{\sin ^{\nu}(\tau) \cdot \sin ^{n-\nu}(1-\tau)}{\sin ^{n}(1)}, \nu=0, \ldots, n .
$$

Both sets of functions form a basis of $\mathcal{T}_{m}$. Moreover, since $l_{\nu}\left(\frac{\mu}{n}\right)=\delta_{\mu \nu}$ for $\mu, \nu=0, \ldots, n$, the trigonometric polynomial (curve)

$$
L(\tau)=\sum_{\nu=0}^{n} d_{\nu} l_{\nu}(\tau)
$$

interpolates the points $d_{0}, \ldots, d_{n}$, whereas the trigonometric Bernstein-Bézier curve

$$
B(\tau)=\sum_{\nu=0}^{n} d_{\nu} b_{\nu}(\tau)
$$

possesses nice shape-preserving properties.

We now make the following construction: With a real parameter $a, 0 \leq a \leq \frac{1}{n}$, and the variable $\tau$ we set

$$
\varphi_{k}(\tau):=\prod_{j=0}^{k-1} \sin (\tau-j a),
$$

for $k \in \mathbb{N}$, and $\varphi_{0} \equiv 1$. Then, for each $\nu \in\{0, \ldots, n\}$, the trigonometric polynomial

$$
s_{\nu}(\tau, a):=\frac{\varphi_{\nu}(\tau, a) \cdot \varphi_{n-\nu}(1-\tau, a)}{\varphi_{\nu}\left(\tau_{a}, a\right) \cdot \varphi_{n-\nu}\left(\tau_{a}, a\right)},
$$

where $\tau_{a}:=1-a(n+1)$, is denoted as $\nu^{\text {th }}$ Stancu polynomial. Obviously, $s_{\nu} \in \mathcal{T}_{m}$. Moreover, the following result holds: 
Theorem 1: a) For each parameter value $0 \leq a \leq \frac{1}{n}$, it is

$$
\mathcal{T}_{m}=\operatorname{span}\left\{s_{0}(\cdot, a), \ldots, s_{n}(\cdot, a)\right\} .
$$

b) For each $\nu \in\{0, \ldots, n\}$, we have

$$
s_{\nu}(\tau, a)= \begin{cases}b_{\nu}(\tau) & \text { for } a=0, \text { and } \\ l_{\nu}(\tau) & \text { for } a=\frac{1}{n} .\end{cases}
$$

Proof. We consider the relation

$$
\sum_{\nu=0}^{n} c_{\nu} s_{\nu}(\tau, a)=0 \text { for } \tau \in[0,1]
$$

In particular, (1.7) must hold for $\tau=0$. Since $s_{\nu}(0, a)=0$ for $\nu=1, \ldots, n$, it follows that $c_{0}$ must be zero. Analogously, setting $\tau=a, 2 a, \ldots,(n-1) a$, it follows successively that $c_{1}=\cdots c_{n}=0$. This proves the linear independence of the functions $s_{\nu}$ and therefore statement a). Statement b) can be verified by straightforward calculation.

Theorem 1 shows in particular that the trigonometric Stancu Curve

$$
S(\tau, a)=\sum_{\nu=0}^{n} d_{\nu} s_{\nu}(\tau, a)
$$

establishes a link between the interpolatory and the shape preserving curves (1.3) and (1.4). The real parameter $a$ should be denoted as design parameter.

\section{Stancu Polynomials over a Triangle}

In this section we transfer the idea described in Section 1 to the case of bivariate trigonometric polynomials over a triangle. So, as a special case, we will also obtain trigonometric triangular Bernstein Bézier polynomials. The corresponding (algebraic) polynomials were introduced and investigated in (Farin, 1986) resp. (Walz, 1991).

As usual, we describe a point $P$ on a given fixed triangle $\left\langle T_{1}, T_{2}, T_{3}\right\rangle$ by its barycentric coordinates $\left(\tau_{1}, \tau_{2}, \tau_{3}\right)$, i.e.,

$$
\begin{aligned}
& P=\tau_{1} T_{1}+\tau_{2} T_{2}+\tau_{3} T_{3}, \\
& 1=\tau_{1}+\tau_{2}+\tau_{3} .
\end{aligned}
$$

We will use the index set

$$
N_{n}:=\left\{\left(\nu_{1}, \nu_{2}, \nu_{3}\right) \in \mathbb{N}_{0}^{3} ; \nu_{1}+\nu_{2}+\nu_{3}=n\right\}
$$

with $\left(\begin{array}{c}n+2 \\ 2\end{array}\right)$ elements. Then, with the auxiliary functions $\varphi_{k}$ and the point $\tau_{a}$ from above, we define for each $\left(\nu_{1}, \nu_{2}, \nu_{3}\right) \in N_{n}$ the Stancu polynomial over a triangle as

$$
s_{\left(\nu_{1}, \nu_{2}, \nu_{3}\right)}(P, a):=\frac{\varphi_{\nu_{1}}\left(\tau_{1}, a\right) \cdot \varphi_{\nu_{2}}\left(\tau_{2}, a\right) \cdot \varphi_{\nu_{3}}\left(\tau_{3}, a\right)}{\varphi_{\nu_{1}}\left(\tau_{a}, a\right) \cdot \varphi_{\nu_{2}}\left(\tau_{a}, a\right) \cdot \varphi_{\nu_{3}}\left(\tau_{a}, a\right)} .
$$


The following theorem, which also can be proved by straightforward calculation, shows that this one-parameter family of functions contains the trigonometric Bernstein polynomials

$$
b_{\left(\nu_{1}, \nu_{2}, \nu_{3}\right)}(P)=\frac{\sin ^{\nu_{1}}\left(\tau_{1}\right) \cdot \sin ^{\nu_{2}}\left(\tau_{2}\right) \cdot \sin ^{\nu_{3}}\left(\tau_{3}\right)}{\sin ^{n}(1)}
$$

as well as the trigonometric Lagrange polynomials

$$
l_{\left(\nu_{1}, \nu_{2}, \nu_{3}\right)}(P)=\prod_{j_{1}=0}^{\nu_{1}-1} \frac{\sin \left(\tau_{1}-\frac{j_{1}}{n}\right)}{\sin \left(\frac{j_{1}+1}{n}\right)} \cdot \prod_{j_{2}=0}^{\nu_{2}-1} \frac{\sin \left(\tau_{2}-\frac{j_{2}}{n}\right)}{\sin \left(\frac{j_{2}+1}{n}\right)} \cdot \prod_{j_{3}=0}^{\nu_{3}-1} \frac{\sin \left(\tau_{3}-\frac{j_{3}}{n}\right)}{\sin \left(\frac{j_{3}+1}{n}\right)}
$$

over triangles as special cases.

Theorem 2: For each $\left(\nu_{1}, \nu_{2}, \nu_{3}\right) \in N_{n}$, we have

$$
s_{\left(\nu_{1}, \nu_{2}, \nu_{3}\right)}(P, a)= \begin{cases}b_{\left(\nu_{1}, \nu_{2}, \nu_{3}\right)}(P) & \text { for } a=0, \text { and } \\ l_{\left(\nu_{1}, \nu_{2}, \nu_{3}\right)}(P) & \text { for } a=\frac{1}{n}\end{cases}
$$

Corollary: For each set of $\left(\begin{array}{c}n+2 \\ 2\end{array}\right)$ points $\left\{d_{\left(\nu_{1}, \nu_{2}, \nu_{3}\right)} ;\left(\nu_{1}, \nu_{2}, \nu_{3}\right) \in N_{n}\right\}$, the surface

$$
L(P):=\sum_{\left(\nu_{1}, \nu_{2}, \nu_{3}\right) \in N_{n}} d_{\left(\nu_{1}, \nu_{2}, \nu_{3}\right)} \cdot l_{\left(\nu_{1}, \nu_{2}, \nu_{3}\right)}(P)
$$

possesses for all $\left(i_{1}, i_{2}, i_{3}\right) \in N_{n}$ the interpolation property

$$
L\left(\frac{i_{1}}{n}, \frac{i_{2}}{n}, \frac{i_{3}}{n}\right)=d_{\left(i_{1}, i_{2}, i_{3}\right)} .
$$

Proof. Follows from the fact that for all $\left(i_{1}, i_{2}, i_{3}\right)$ and $\left(\nu_{1}, \nu_{2}, \nu_{3}\right) \in N_{n}$,

$$
l_{\left(\nu_{1}, \nu_{2}, \nu_{3}\right)}\left(\frac{i_{1}}{n}, \frac{i_{2}}{n}, \frac{i_{3}}{n}\right)= \begin{cases}1, & \text { if }\left(i_{1}, i_{2}, i_{3}\right)=\left(\nu_{1}, \nu_{2}, \nu_{3}\right) \\ 0, & \text { otherwise }\end{cases}
$$

\section{References}

Alfeld, P., Neamtu, M. \& Schumaker, L.L. (1995), Circular Bernstein-Bèzier Polynomials, in: Mathematical Methods for Curves and Surfaces, M. Daehlen, T. Lyche \& L.L.Schumaker eds., Vanderbilt University Presss, Nashville, $11-20$.

Farin, G. (1986), Triangular Bernstein-Bézier Patches, Computer Aided Geom. Design 3, 83 127.

Farin, G. \& Barry, P.J. (1986), Link between Bézier and Lagrange Curve and Surface Schemes, Computer-Aided Design 18, 525 - 528. 
Gonsor, D. \& Neamtu, M. (1996), Null Spaces of Differential Operators, Polar Forms and Splines, J. Approx. Theory 86, $81-107$.

Koch, P.E., Lyche, T., Neamtu, M. \& Schumaker, L.L. (1995), Control Curves and Knot Insertion for Trigonometric Splines, Adv. in Comp. Maths. 3, $405-424$.

Peña, J.M. (1996), Shape Preserving Representations for Trigonometric Polynomial Curves, Preprint, University of Zaragoza.

Walz, G. (1988), On Generalized Bernstein Polynomials in CAGD, Mannh. Math. Manuskr. 86. Walz, G. (1991), Spline-Funktionen im Komplexen, B.I.-Wissenschaftsverlag, Mannheim/ Wien/Zürich.

Walz, G. (1997), Some Identities for Trigonometric B-Splines, with Application to Curve Design, to appear in BIT.

Guido Walz

Fakultät für Mathematik und Informatik

Universität Mannheim

D-68131 Mannheim

Germany

walz@math.uni-mannheim.de 
.

-

. 